\title{
Diagnóstico diferencial del megaprepucio congénito: relevancia terapéutica y pronóstica
}

\author{
J. Arredondo Montero, Y. González Ruiz, J.V. Redondo Sedano, S. Hernández Martín, L. Ayuso González, C. Bardají Pascual \\ Complejo Hospitalario de Navarra. Pamplona, Navarra.
}

\begin{abstract}
RESUMEN
El megaprepucio congénito es una patología urológica propia de la infancia que puede ser fácilmente confundida con otras entidades clínicas como la fimosis fisiológica o el pene enterrado. Debido al riesgo de complicaciones asociadas, principalmente de carácter infeccioso $\mathrm{u}$ obstructivo con afectación del tracto urinario superior, es importante incidir en su correcto diagnóstico de cara a ofertar un tratamiento precoz. Presentamos tres casos de megaprepucio congénito, diagnosticados e intervenidos en nuestro servicio durante el periodo comprendido entre enero de 2019 y mayo de 2020, describiéndose el diagnóstico, la terapéutica empleada y la evolución clínica.
\end{abstract}

Palabras Clave: Megaprepucio congénito; Fimosis; Pene enterrado; Balonización; Prepucioplastia; Circuncisión.

DIFFERENTIAL DIAGNOSIS OF CONGENITAL MEGAPREPUCE: THERAPEUTIC AND PROGNOSTIC RELEVANCE

\begin{abstract}
Congenital megaprepuce is a urological pathology typical of childhood. It can be easily mistaken for other clinical entities such as physiological phimosis or buried penis. Owing to the risk of associated complications - primarily infectious and obstructive complications, with upper urinary tract involvement -, achieving an accurate diagnosis proves particularly significant for early treatment initiation. We present three cases of congenital megaprepuce diagnosed and operated on at our department from January 2019 to May 2020. Diagnosis, therapy, and clinical progression are described.
\end{abstract}

KeY Words: Megaprepuce; Congenital; Phimosis; Penis; Buried; Ballooning, Preputioplasty; Circumcision; Technique.

DOI: $10.54847 /$ cp.2022.01.10

Correspondencia: Dr. Javier Arredondo Montero.

E-mail: Javier.montero.arredondo@gmail.com

Recibido: Septiembre 2020

Aceptado: Febrero 2021
El megaprepucio congénito es una patología infrecuente caracterizada por una redundancia de mucosa prepucial acompañada de un defecto parcial de piel prepucial ventral, que se encuentra engrosada. Esta situación provoca una cobertura completa del pene, sin existir, en principio, otras alteraciones peneanas asociadas ${ }^{(1)}$. La presentación clínica más habitual es la balonización prepucial por retención urinaria. El diagnóstico de esta patología es clínico ${ }^{(2)}$, no siendo preciso complementar con pruebas adicionales salvo en casos en los que se sospeche coexistencia de otras patologías genitourológicas.

El conocimiento del megaprepucio congénito es relevante por varios motivos: por la frecuente asociación de infecciones de tracto urinario, por la implicación estética que presenta el cuadro y por el hecho de que el manejo quirúrgico difiere del aplicado en la fimosis convencional.

El diagnóstico diferencial del megaprepucio congénito incluye fundamentalmente dos entidades clínicas: la fimosis fisiológica y el pene enterrado.

La fimosis fisiológica, causada por la separación incompleta de las capas epiteliales del glande y del prepucio y por la propia forma cónica del glande, es una entidad frecuente que afecta hasta al 96\% de los recién nacidos y que en la gran mayoría de los casos resuelve de forma espontánea antes de los 4 años de edad. El manejo inicial de esta entidad suele ser conservador, con corticoterapia tópica y medidas higiénicas, reservándose la cirugía (prepucioplastia o circuncisión) para los casos complicados (balanopostitis de repetición, infecciones de tracto urinario) o refractarios a tratamiento médico.

El pene enterrado es una malformación perteneciente al espectro de las anomalías de la fusión penoescrotal. Se caracteriza por un pene anclado a la fascia profunda mediante uniones anormales del dartos, y por una unión de escroto y piel que se extiende distalmente, lo que visualmente provoca la errónea impresión de tratarse de un micropene. Existen múltiples propuestas para la corrección quirúrgica del pene enterrado, como por ejemplo la técnica de Maizels. No obstante, existe consenso en considerar el megaprepucio congénito como una entidad distinta al pene enterrado que precisa un manejo quirúrgico específico. 


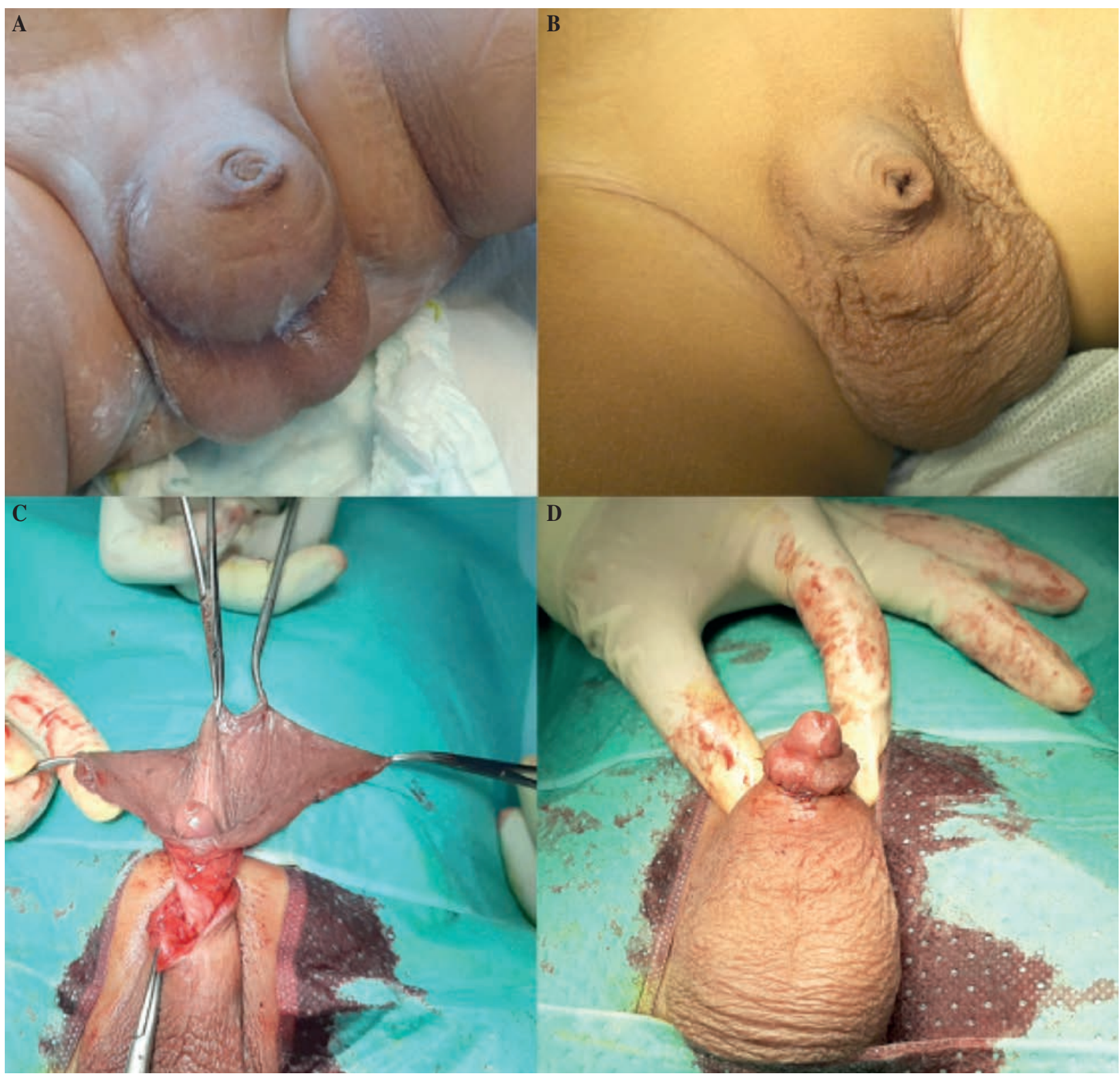

Figura 1. Caso clínico 1. A) Balonización por acúmulo urinario en espacio balanoprepucial. B) Situación basal del paciente de forma previa a la intervención. C) Exposición de la redundancia mucosa característica del megaprepucio congénito. D) Resultado postquirúrgico inmediato.

Se presentan tres casos de megaprepucio congénito diagnosticados y tratados quirúrgicamente en nuestro centro. En los dos primeros casos la cirugía inicial se llevó a cabo antes del año de vida, con la finalidad de corregir la balonización y evitar las complicaciones asociadas a la patología. En el tercer caso se realizó la cirugía correctora en un único tiempo a los 17 meses de vida.

En todos los pacientes se evidenció durante la valoración inicial una balonización prepucial por retención de orina secundaria a la estrechez del orificio prepucial. Ambos pacientes habían presentado infecciones de tracto urinario de forma previa a nuestra valoración.
El primer paciente presentaba el antecedente médico de una pielonefritis. Se realizó el diagnóstico de fimosis puntiforme cicatricial con balonización prepucial llamativa asociada y, tras objetivarse refractariedad al tratamiento médico con corticoterapia tópica, se indicó la realización de una circuncisión reglada. Durante dicha intervención se evidenció una redundancia llamativa de la mucosa prepucial, que se resecó y se reconstruyó mediante una sutura reabsorbible de la corona mucocutánea según la técnica habitual empleada en las circuncisiones en nuestro centro (Fig. 1). En el postoperatorio inmediato dicho paciente presentó un enterramiento progresivo del pene en la región escrotal, que 


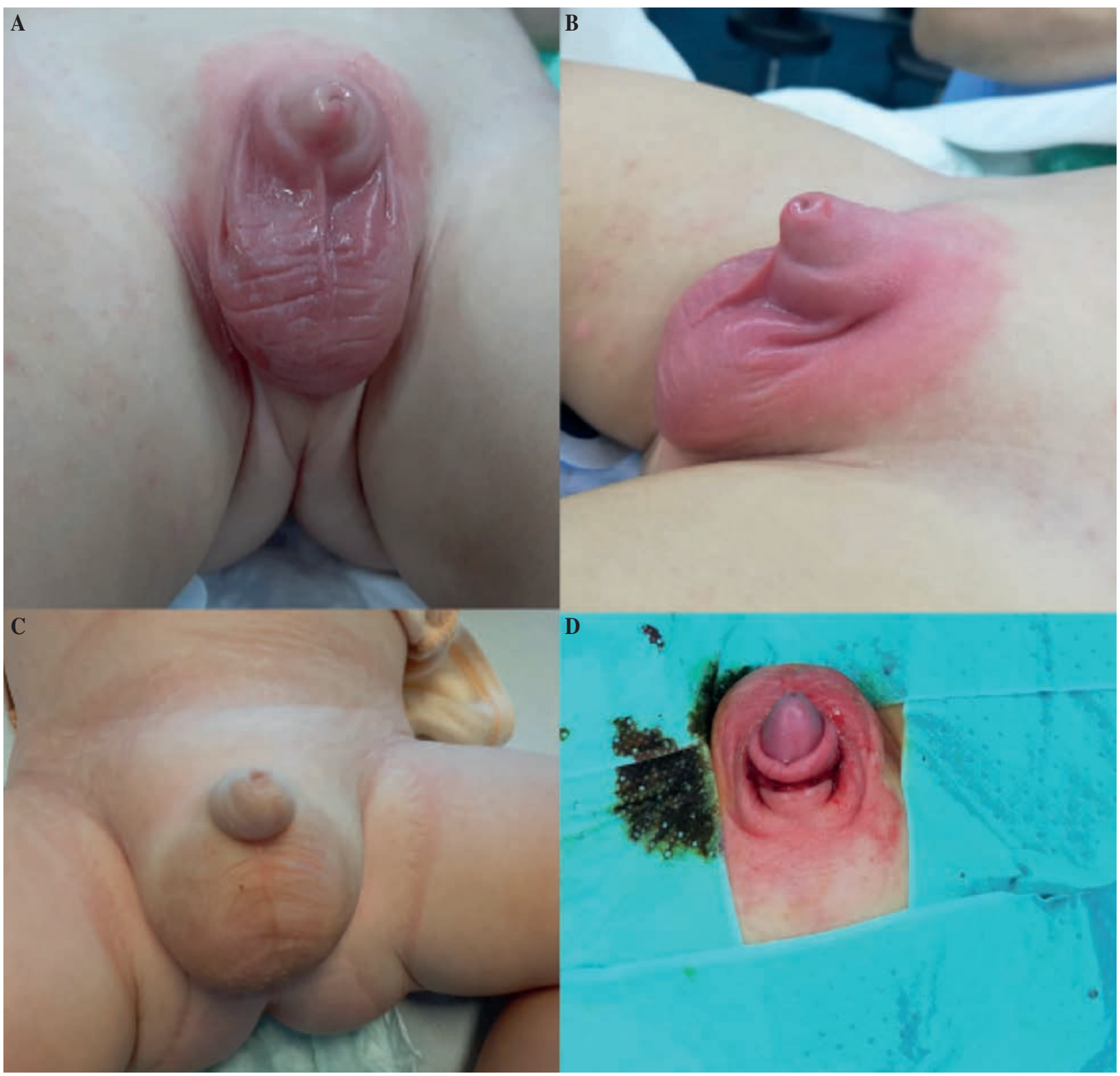

Figura 2. Caso clínico 2. A) Situación basal del paciente, con eritema perigenital evidente. B) Visión lateral. C) Balonización por acúmulo urinario en espacio balanoprepucial. D) Resultado postquirúrgico inmediato.

se manejó con corticoterapia y vigilancia evolutiva. Dicho enterramiento mejoró progresivamente durante los primeros días postoperatorios.

El segundo paciente presentaba el antecedente médico de infecciones urinarias de repetición. En la exploración física se evidenció estrechez prepucial con balonización asociada y con importante eritema perigenital en probable relación con irritación química por fuga urinaria. Se realizó el diagnóstico de megaprepucio congénito y se indicó la realización de una prepucioplastia según la técnica de Duhamel como técnica puente hasta la corrección definitiva (Fig. 2). Dicho paciente presentó una evolución postoperatoria favorable.
El tercer paciente presentaba el antecedente médico de una infección urinaria aislada. En la exploración física se evidenció estrechez prepucial con balonización. Se realizó el diagnóstico de megaprepucio congénito y se indicó la realización de una corrección quirúrgica siguiendo la técnica conocida como "reconstrucción anatómica" (anatomical approach) (Fig. 3). Durante dicha intervención se evidenciaron lesiones ulcerativas distribuidas de forma difusa por el glande. Dicho paciente presentó una evolución postoperatoria inmediata favorable.

Los tres pacientes fueron revisados en consultas externas al mes y a los seis meses de la intervención quirúrgica, sin 


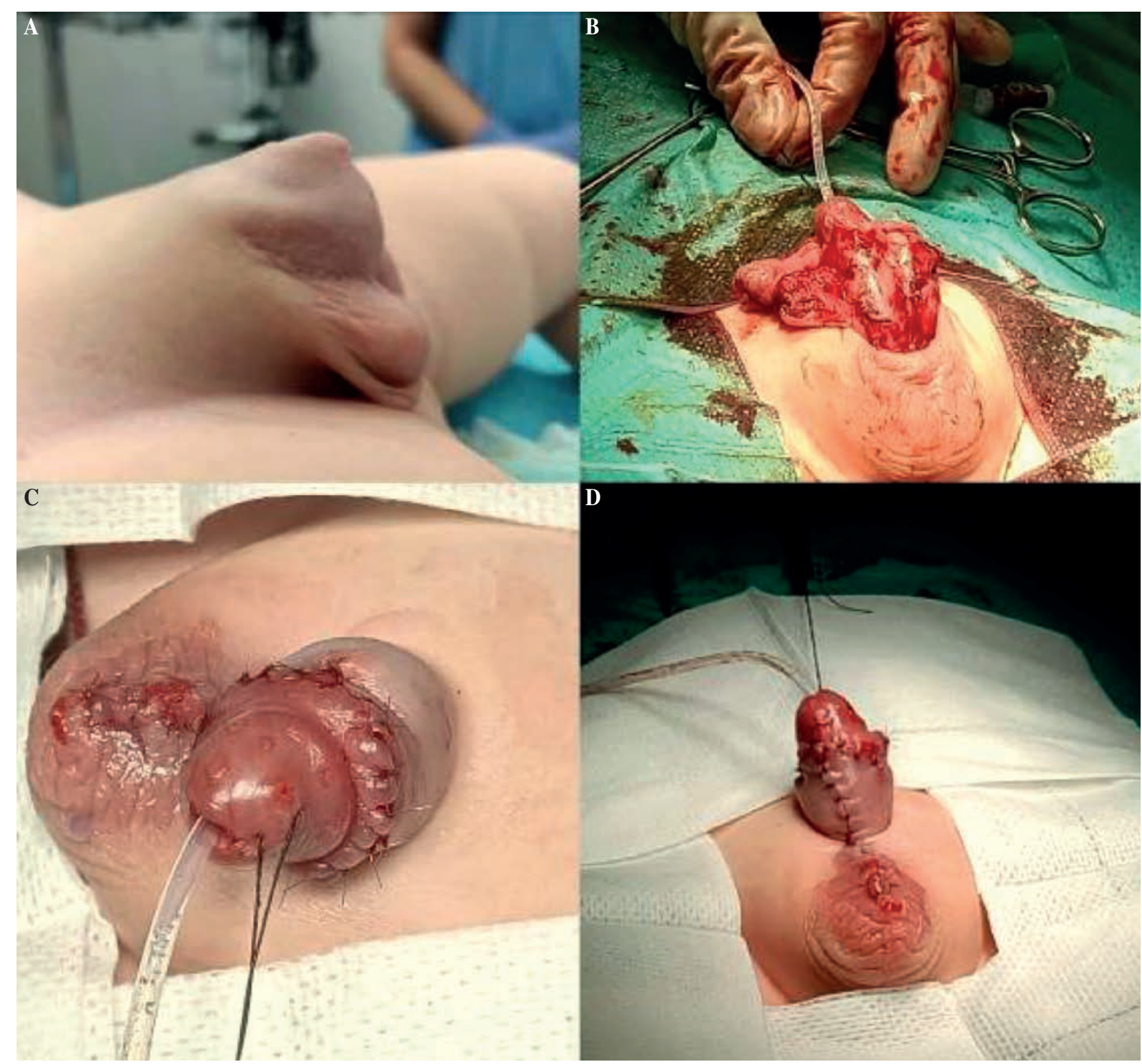

Figura 3. Caso clínico 3. A) Situación basal del paciente, con balonización evidente. B) Corrección quirúrgica mediante la técnica "anatomical approach". C) Lesiones de carácter ulcerativo evidenciadas en el glande tras su exposición durante la cirugía. D) Resultado postquirúrgico inmediato.

evidenciarse complicaciones postoperatorias. En todos los pacientes se objetivó una mejoría funcional importante, normalizándose la micción y corrigiéndose la balonización prepucial presente de forma previa a la intervención. El aspecto estético del pene difirió, presentando el segundo y el tercer paciente unas evoluciones estéticas precoces más satisfactorias que el paciente sometido a una circuncisión reglada (Fig. 4).

\section{COMENTARIOS}

El megaprepucio congénito es una patología infrecuente y que puede ser fácilmente confundida con otras patologías como la fimosis fisiológica o el pene enterrado. Dado que la presentación clínica suele ser sintomática (con balonización prepucial) y dado que no es infrecuente la asociación con infecciones del trato urinario o la afectación cutánea local por irritación química, existe indicación de manejo quirúrgico precoz - al contrario que en el caso de la fimosis en pacientes de este rango etario, en los que se considera fisiológica-. Al tratarse de una patología con implicaciones terapéuticas y quirúrgicas propias, consideramos importante conocer su existencia a la hora de realizar el diagnóstico diferencial de una alteración peneana en la primera infancia. Su corrección quirúrgica presenta multitud de técnicas descritas: la V-plastia ventral, la genitoplastia con separación penoescrotal, la técnica 

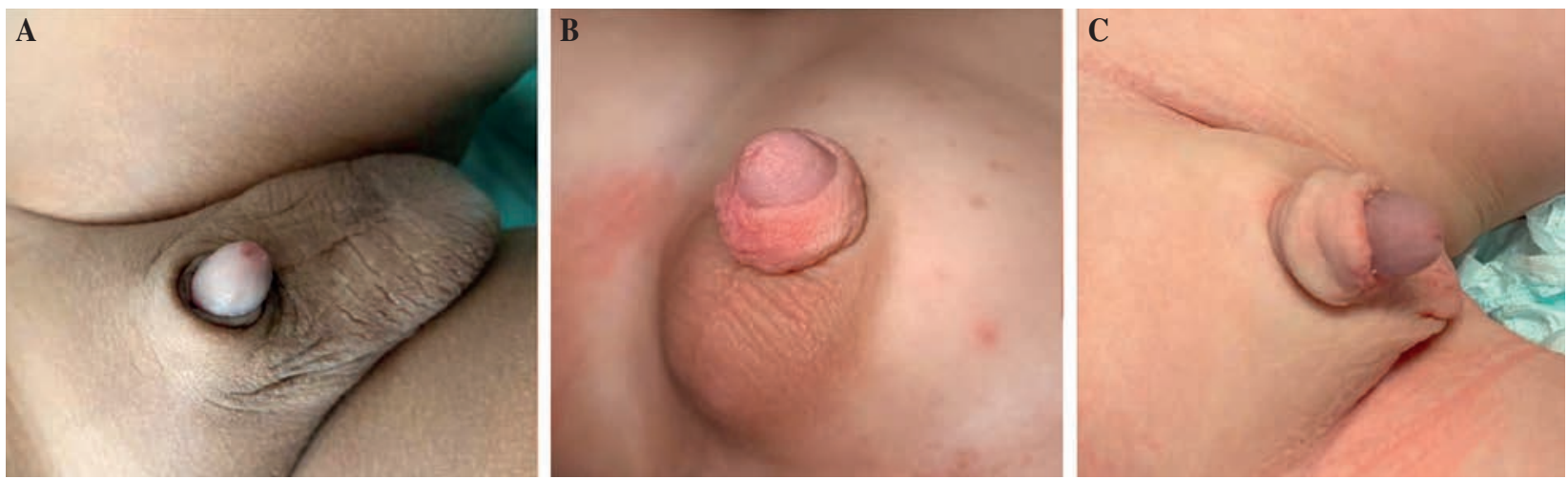

Figura 4. Evolución postquirúrgica a los 6 meses de la intervención. A) Paciente 1. B) Paciente 2. C) Paciente 3.

de doble incisión longitudinal (DOLOMITE), el abordaje con incisión en arco o la reconstrucción anatómica ${ }^{(3-6)}$.

En la actualidad existe poca literatura en relación con el seguimiento a medio y largo plazo de estos pacientes, siendo necesarios estudios adicionales.

\section{BIBLIOGRAFÍA}

1. Werner Z, Hajiran A, Al-Omar O. Congenital Megaprepuce: Literature Review and Surgical Correction. Case Rep Urol. 2019; 2019: 1-6.

2. Delgado Oliva FJ, Domínguez Hinarejos C, Serrano Durbá S, Estornell Moragues F, Martínez Verduch M, García Ibarra F. Megaprepucio congénito: diagnóstico y manejo terapéutico. Actas Urol Esp [Internet]. 2006 [citado 2019 Dic 25]; 30(10): 1038-42.
3. Alexander A, Lorenzo AJ, Salle JLP, Rode H. The Ventral V-plasty: A simple procedure for the reconstruction of a congenital megaprepuce. J Pediatr Surg [Internet]. 2010; 45(8): 1741-7.

4. Callewaert PRH, Rahnama'I MS, Guimarães MNC, Vrijens DMJ, Van Kerrebroeck PEVA. DOuble LOngitudinal megapreputium incision TEchnique: The Dolomite. Urology [Internet]. 2014; 83(5): 1149-54.

5. Lin HW, Zhang L, Geng HQ, Fang XL, Xu GF, Xu MS, et al. An arc incision surgical approach in congenital megaprepuce. Chin Med J (Engl). 2015; 128(4): 555.

6. Lasso Betancor CE, Cherian A, Smeulders N, Mushtaq I, Cuckow P. Mid- to long-term outcomes of the 'anatomical approach' to congenital megaprepuce repair. J Pediatr Urol. 2019; 15(3): 243. e1-243.e6. 